\title{
Remembering the laser diode
}

\author{
Fifty years ago, researchers at a handful of laboratories around the world were reporting lasing from \\ the first semiconductor lasers. Our IT infrastructure today relies on their diligence and success.
}

In 2010, the 50th anniversary of

Ted Maiman's experimental demonstration of the first ruby laser quite rightly gave rise to substantial publicity, including celebratory events such as the LaserFest series (www.laserfest.org) and much coverage in a wide variety of newspapers and magazines. However, in many ways it was actually the successful development of the GaAs semiconductor laser diode in 1962 that really ignited the laser revolution. The fabrication of a semiconductor laser kick-started the important field of optoelectronics and showed that lasers could, in principle, be fabricated in a miniature, cost-effective format that showed great promise for mass-production in a form similar to electronic devices. Shortly afterwards, the demonstration of electrically pumped devices capable of operating at room-temperature with high output powers further added to the practicality and appeal of semiconductor lasers.

In contrast, if lasers had remained limited to getting their gain from doped glass crystals such as ruby or Ti:sapphire, or gas mixtures such as $\mathrm{HeNe}$ or $\mathrm{CO}_{2}$, their technological impact would have been considerably less and the world of photonics would look very different. Although the use of high-power lasers for materials processing applications such as cutting and welding might still have come to fruition, many other tasks that we take for granted today would probably not have been feasible. Take, for example, optical data storage and communication networks, which today represent two of largest and most important markets for laser technology. It is unlikely that fibre-based data networks, CDs, DVDs and BluRay players would have been seriously pursued or even considered practical without the realization of an efficient and low-cost laser diode.

Given this context, it is pleasing to see that the 50th birthday of the laser diode has not been overlooked. The Institute of Physics in the UK organized a two-day celebratory seminar entitled " 50 th Anniversary of the Laser Diode" at the University of Warwick on 20-21 September 2012, during which several of the founding fathers of the technology reminisced about the early days of its development and the race to make the first laser diode. Speakers included Marshall Nathan, formerly of IBM Labs in

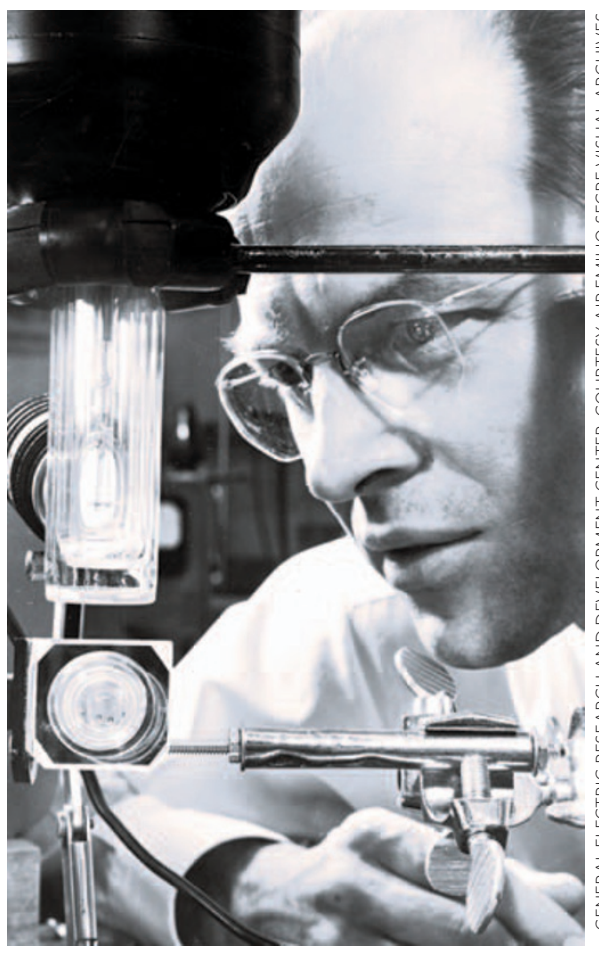

Applied Physics Letters by Marshall Nathan's group at IBM's Watson Research Labs ${ }^{2}$ and Ted Quist's group from the Massachusetts Institute of Technology Lincoln Labs ${ }^{3}$. A flurry of results then followed from around the world, including the first visible semiconductor laser by Nick Holonyak's group at General Electric's lab in Syracuse (who became famous for inventing the light-emitting diode that same year) as well as demonstrations by Cyril Hilsum and colleagues at the UK's Services Electronics Research Laboratory and a Russian group of scientists led by Nikolay Basov.

Speaking at the event, Marshall Nathan says that in early 1962 he was at IBM studying the photoluminescence from GaAs when the department director Rolf Landauer announced that he wanted IBM to be the first group to make a GaAs laser and threw a team of people onto the project. In February, Nathan and his co-workers tried using a flash lamp to pump a sample of GaAs but were unsuccessful in achieving lasing. Then, following further research in September 1962, they observed "spectacular line narrowing", which suggested that lasing was taking place.

"We sent the results to Applied Physics the USA, Yuri Popov from Russia, Maurice Bernard from France, Jim Coleman from the USA and Cyril Hilsum, Robin Thompson and John Orton from the UK.

The competition between various scientists (such as Gordon Gould, Charles Townes, Art Schawlow and Ted Maiman, among others) to achieve the first experimental demonstration of a laser in 1960 is well documented (see the excellent account in Jeff Hecht's book "Beam: The Race to Make the Laser"). However, the quest to develop a semiconductor laser two years later was arguably just as exciting, fastpaced and competitive, with a handful of research groups all reported working lasers based on GaAs $\mathrm{p}-\mathrm{n}$ junctions within a short period in the autumn of 1962.

Ultimately, Robert Hall (pictured) and his group at General Electric's research labs in Schenectady is credited as getting there first. Their report of coherent infrared $(850 \mathrm{~nm})$ emission from a GaAs laser appeared in the 1 November 1962 issue of Physical Review Letters ${ }^{1}$. This was simultaneously accompanied by reports of lasing from GaAs devices appearing in the journal Letters, hand-delivered," said Nathan. "We then started to get lots of results very quickly, such as continuous-wave lasing at $4.2 \mathrm{~K}$, room-temperature lasing and measurement of far-field emission patterns," said Nathan. "The first real semiconductor laser at IBM was made in October 1962, and we got the patent out in just five days."

Astonishingly, Nathan says at the time that the researchers at IBM didn't know that they had serious competition from General Electric and were shocked to learn Hall's APL paper had been submitted 11 days before their own, effectively scooping them. "We had no idea that General Electric was working on this," commented Nathan. This story is not only an interesting history lesson, but also a valuable reminder of just how exciting and fast-paced research can be.

\footnotetext{
References

1. Hall, R N, Fenner, G. E, Kingsley, J. D, Soltys, T. J and Carlson R. O. Phys. Rev. Lett. 9, 366-369 (1962).

2. Nathan M,I, Dumke W.P, Burns G, Dill F, H, Jr and Lasher G Appl. Phys. Lett. 1, 62 (1962).

3. Quist T.M et al Appl. Phys. Lett. 1, 91 (1962).
} 